\title{
Anatomical Location of Interest
}

National Cancer Institute

\section{Source}

National Cancer Institute. Anatomical Location of Interest. NCI Thesaurus. Code C161481.

The identification of an anatomical location that may contain a tumor, lesion or site of disease, and is part of the assessment of the response to the therapeutic intervention. 